\title{
Accelerated hyperfractionation plus temozolomide in glioblastoma
}

\author{
David Kaul ${ }^{*}$, Julian Florange ${ }^{\dagger}$, Harun Badakhshi, Arne Grün, Pirus Ghadjar, Sebastian Exner and Volker Budach
}

\begin{abstract}
Introduction: Hyperfractionated (HFRT) or accelerated hyperfractionated radiotherapy (AHFRT) have been discussed as a potential treatment for glioblastoma based on a hypothesized reduction of late radiation injury and prevention of repopulation. HFRT and AHFRT have been examined extensively in the pre-Temozolomide era with inconclusive results. In this study we examined the role of accelerated hyperfractionation in the Temozolomide era.

Materials and methods: Sixty-four patients who underwent AHFRT (62 of which received Temozolomide) were compared to 67 patients who underwent normofractionated radiotherapy (NFRT) (64 of which received TMZ) between 02/2009 and 10/2014. Follow-up data were analyzed until 01/2015.

Results: Median progression-free survival (PFS) was 6 months for the entire cohort. For patients treated with NFRT median PFS was 7 months, for patients treated with AHFRT median PFS was 6 months. Median overall survival (OS) was 13 months for all patients. For patients treated with NFRT median OS was 15 months, for patients treated with AHFRT median OS was 10 months. The fractionation regimen was not a predictor of PFS or OS in univariable- or multivariable analysis. There was no difference in acute toxicity profiles between the two treatment groups.

Conclusions: Univariable and multivariable analysis did not show significant differences between NFRT and AHFRT fractionation regimens in terms of PFS or OS. The benefits are immanent: the regimen does significantly shorten hospitalization time in a patient collective with highly impaired life expectancy. We propose that the role of AHFRT + TMZ should be further examined in future prospective trials.
\end{abstract}

\section{Introduction}

Gliomas are the most common primary tumors of the central nervous system (CNS) in adults representing about one third of central nervous system tumors and $81 \%$ of all malignant CNS tumors reported in the United States [1]. The most common and most malignant type of glioma is glioblastoma (GBM), with a median overall survival (OS) rate of 15 months after surgical resection followed by adjuvant radiotherapy (RT) and Temozolomide (TMZ) chemotherapy. The prevalence of GBM is highest in patients aged 50 years or older and is likely to increase with the ongoing demographic shift toward older ages [2].

Well-known postitive prognostic factors for OS in GBM patients are young age at diagnosis, high Karnofsky performance score (KPS), great extent of neurosurgical

\footnotetext{
* Correspondence: david.kaul@charite.de

'Equal contributors

Klinik für Radioonkologie und Strahlentherapie, Charité Universitätsmedizin Berlin, Campus Virchow-Klinikum, Augustenburger Platz 1, 13353 Berlin, Germany
}

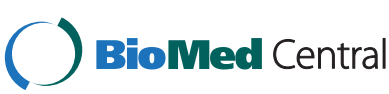

resection, O-6-methylguanine-DNA methyltransferase- gene (MGMT) methylation as well as isocitrate dehydrogenase (IDH) 1-mutational status [3-5]. Current standard of care for newly diagnosed GBM comprises maximal safe resection, adjuvant radiotherapy with (RT) with concurrent TMZ and post-RT TMZ chemotherapy [6, 7]. Fractionated RT to the tumor bed in 30 fractions of 2 Gy in single doses of 2 Gy to a total accumulated dose of 60 Gy delivered over the course of 6 weeks has been widely accepted as the standard fractionation regimen, balancing effectiveness with radiation toxicity. Recently some authors have suggested hypofractionated regimens for the elderly and frail patient population $[8,9]$ other authors have evaluated the role of hypofractionation plus TMZ [10].

Other authors have examined the potential role of hyperfractionated- (HFRT) and accelerated hyperfractionated radiotherapy (AHFRT) as well as the role of protons in GBM [11]. The use of HFRT and AHFRT is based on a hypothesized reduction of late radiation injury and prevention of tumor repopulation in treatment intervals [12]. 
Despite plausible rationales, various trials have failed to prove the superiority of dose-escalated HFRT and AHFRT in the pre-TMZ era [13].

In 1994, the European Organization for the Research and Treatment of Cancer (EORTC) reported an AHFRT dose escalation trial using doses of 42-60 Gy in 2 Gy fractions three times daily, which failed to show differences in survival in all groups. No additional chemotherapy was used [13]. In 1999 Lutterbach et. al. showed survival rates for 1.5 Gy thrice daily to 54 Gy comparable to conventional RT, again no chemotherapy was used [14]. In 2001 Prados et. al. showed data for AHFRT with or without difluromethylornithine (DFMO) vs. conventional irradiation with or without DFMO with no OS benefit for the experimental groups [15].

The RTOG 83-02 study tested HFRT $(2 \times 1.2$ Gy to doses of $64.8,72,76.8$, or $81.6 \mathrm{~Gy})$ vs. AHFRT $(2 \times 1.6 \mathrm{~Gy}$ to doses of 48 or $54.4 \mathrm{~Gy}$ ), all groups received concurrent bis-chloroethyl (BCNU). Contrary to the other aforementioned studies HFRT patients who had received higher doses of 76.8 and 81.6 Gy showed superior survival compared to the AHFRT groups [16].

In summary, the data on HFRT and AHFRT mainly stem from the pre-TMZ era and are not fully conclusive. We therefore want to present experience from our institution on the treatment of patients with newly diagnosed GBM with AHFRT of $2 \times 1.6$ Gy to 59,2 Gy and concurrent and sequential Temozolomide following the Stupp regimen. Apart from a potential reduction of tumor repopulation as well as a hypothesized reduced late toxicity rate, the regimen does significantly shorten hospitalization time in a group of patients with highly impaired life expectancy.

\section{Materials and methods}

Treatment decisions, patient selection and dose regimens Starting from 01/2009 patients with resected GBM with organs-at-risk (OAR) in close proximity to the resection cavity were offered adjuvant radio-chemotherapy (RCTx) with single doses of 1.6 Gy twice daily to a total dose of 59.2 Gy (19 days schedule) as an alternative to a conventional fractionation with single doses of 2 Gy up to $60 \mathrm{~Gy}$ (30 days schedule, NFRT). Of 131 patients 126 received continuous daily TMZ (75 mg per square meter of bodysurface area per day, 7 days per week from the first to the last day of radiotherapy), followed by six cycles of adjuvant TMZ (150 mg per square meter for 5 days during each 28-day cycle).

In this study we carried out a retrospective analysis of 64 patients who underwent AHFRT plus TMZ and compared them with 67 patients who underwent NFRT plus TMZ between $02 / 2009$ and 10/2014. Follow-up data were analyzed until 01/2015.

In our institution treatment decisions are based on the votes of an interdisciplinary tumor board. Usually all patients $<70$ years with a KPS $>50 \%$ are offered adjuvant AHFRT + TMZ or NFRT + TMZ. AHFRT + TMZ is offered when OARs such as the optic nerves, chiasm or brainstem would be touched by the CTV and covered by the PTV, and in case that the patient is willing and fit enough to undergo treatment twice daily.

Patients $\geq 70$ yeas of age either receive hypofractionated radiotherapy or TMZ only (depending on MGMT-status).

\section{Stratification, variables and follow-up}

Patients were stratified according to fractionation scheme, age, gender, KPS, extent of surgery (biopsy, partial-, gross total resection), MGMT-status, tumor localization (frontal, parietal, temporal, occipital, central) and planning target volume (PTV). Follow-up examinations, including MRI as well as clinical and neurologic examinations were performed at 6-8 week intervals after radiotherapy.

\section{Treatment planning}

Target delineation in GBM varies substantially between different institutions and several consensus statements are available. However, an ESTRO-ACROP guideline is available since January 2016 [17]. Adjuvant RCTx was initiated within 4 weeks after surgical resection or stereotactic biopsy. Contrast agent enhanced computed tomography in a thermoplastic mask as well as gadolinium enhanced magnetic resonance imaging (MRI) was performed before RT planning.

Target volumes were based on preoperative and postoperative MRI. The gross tumor volume (GTV) was defined as the summation of the postoperative surgical cavity with or without residual tumor lesion(s) as well as tumor extension on the preoperative T1-weighted gadoliniumenhanced imaging. The diffusion-weighted imaging (DWI) images were also used in the estimation of GTV. The extent of peritumoral edema was not routinely included in the clinical target volume (CTV), however, an all-round GTV margin of $2 \mathrm{~cm}$ was mandatory. For the planning target volume (PTV) an additional $0.5 \mathrm{~cm}$ margin was added. Intensity-modulated radiation therapy (IMRT) was applied using a 6-MV linear accelerator with multileaf collimators. Until 2012 treatment was performed using step-and-shoot intensity-modulated radiation therapy (IMRT), starting in early 2012 all patients were treated using volumetric arc therapy (VMAT).

\section{Toxicity}

Higher grade acute toxicity $\left(\geq 3^{\circ}\right)$ was analyzed for 90 days post treatment according to CTCAE 4.0.

\section{Formulas and statistics}

Overall survival (OS) and progression-free survival (PFS) were calculated from the first day of irradiation 
using Kaplan-Meier analysis and the log-rank test. Progression was defined retrospectively by clinical note assessments that included integration of imaging and clinical status. Subgroups were compared using univariable analysis and the Cox proportional hazard model for multivariable analysis. A p-value of less than 0.05 was considered statistically significant. A $p$-value of less than 0.1 was considered a trend. All variables from the univariable analysis were included in multivariable analysis. All statistical analyses were performed using IBM SPSS Statistics 19 (New York, USA).

\section{Results}

Patient characteristics

Patient characteristics are shown in Table 1. One hundred thirty-one patients treated for GBM were identified in our retrospective analysis. Sixty-seven were treated with NFRT and 64 patients were treated using AHFRT.

The two groups were well matched in terms of gender, PTV, tumor localization, MGMT-status, extent of surgery, KPS and TMZ treatment and salvage treatment. Median age in the AHFRT group was lower than in the NFRT group $(p<0.001)$.

Table 1 Patient characteristics of the 131 GBM patients analyzed

\begin{tabular}{|c|c|c|c|c|c|c|c|c|}
\hline \multirow{3}{*}{\multicolumn{2}{|c|}{$\overline{\text { Median Age (min/max) [y] }}$}} & \multirow{2}{*}{\multicolumn{2}{|c|}{$\frac{\text { Overall Collective }}{(n=131)}$}} & \multirow{2}{*}{\multicolumn{2}{|c|}{$\frac{N F R T}{(n=67)}$}} & \multirow{2}{*}{\multicolumn{2}{|c|}{$\frac{\text { AHFRT }}{(n=64)}$}} & \multirow{3}{*}{$\begin{array}{l}p \text {-value } \\
p<0.001\left(^{*}\right)\end{array}$} \\
\hline & & & & & & & & \\
\hline & & 61 & $12 / 80$ & 63 & $43 / 78$ & 59 & $12 / 80$ & \\
\hline \multirow{2}{*}{\multicolumn{2}{|c|}{ Mean PTV \pm sd $[\mathrm{ccm}]$}} & 355 & \pm 142 & 339 & \pm 141.4 & 373 & \pm 141.8 & $p=0.17$ \\
\hline & & $\mathrm{n}$ & $\%$ & $n$ & $\%$ & $n$ & $\%$ & \\
\hline \multirow[t]{2}{*}{ Gender } & $\mathrm{m}$ & 88 & $67.2 \%$ & 46 & $68.7 \%$ & 42 & $65.6 \%$ & $p=0.85$ \\
\hline & $f$ & 43 & $32.8 \%$ & 21 & $31.3 \%$ & 22 & $34.4 \%$ & \\
\hline \multirow[t]{6}{*}{ Localization } & Frontal & 42 & $32.1 \%$ & 16 & $23.9 \%$ & 26 & $40.6 \%$ & $p=0.38$ \\
\hline & Parietal & 31 & $23.7 \%$ & 17 & $25.4 \%$ & 14 & $21.9 \%$ & \\
\hline & Temporal & 38 & $29.0 \%$ & 22 & $32.8 \%$ & 16 & $25.0 \%$ & \\
\hline & Occipital & 9 & $6.9 \%$ & 4 & $6.0 \%$ & 5 & $7.8 \%$ & \\
\hline & Central & 9 & $6.9 \%$ & 6 & $9.0 \%$ & 3 & $4.7 \%$ & \\
\hline & $\mathrm{n} / \mathrm{a}$ & 2 & $1.5 \%$ & 2 & $3.0 \%$ & 0 & $0.0 \%$ & \\
\hline \multirow[t]{3}{*}{ MGMT-status } & unmethylated & 63 & $48.1 \%$ & 32 & $47.8 \%$ & 31 & $48.4 \%$ & $p=0.66$ \\
\hline & methylated & 43 & $32.8 \%$ & 23 & $34.3 \%$ & 20 & $31.3 \%$ & \\
\hline & $\mathrm{n} / \mathrm{a}$ & 25 & $19.1 \%$ & 12 & $17.9 \%$ & 13 & $20.3 \%$ & \\
\hline \multirow[t]{4}{*}{ Extent of surgery } & Biopsy & 16 & $12.2 \%$ & 6 & $9.0 \%$ & 10 & $15.6 \%$ & $p=0.38$ \\
\hline & Partial resection & 57 & $43.5 \%$ & 28 & $41.8 \%$ & 29 & $45.3 \%$ & \\
\hline & Gross tumor resection & 51 & $38.9 \%$ & 29 & $43.3 \%$ & 22 & $34.4 \%$ & \\
\hline & $\mathrm{n} / \mathrm{a}$ & 7 & $5.3 \%$ & 4 & $6.0 \%$ & 3 & $4.7 \%$ & \\
\hline \multirow[t]{4}{*}{ KPS } & $50 \%$ & 7 & $5.3 \%$ & 4 & $6 \%$ & 3 & $4.7 \%$ & $p=0.3$ \\
\hline & $60 \%$ & 49 & $37.4 \%$ & 27 & $40 \%$ & 22 & $34.4 \%$ & \\
\hline & $70 \%$ & 47 & $35.9 \%$ & 24 & $36 \%$ & 23 & $35.9 \%$ & \\
\hline & $80 \%$ & 28 & $21.4 \%$ & 12 & $18 \%$ & 16 & $25.0 \%$ & \\
\hline \multirow[t]{2}{*}{ Temozolomide } & yes & 126 & $96.2 \%$ & 65 & $97.0 \%$ & 61 & $95.3 \%$ & $p=0.68$ \\
\hline & no & 5 & $3.8 \%$ & 2 & $3.0 \%$ & 3 & $4.7 \%$ & \\
\hline \multirow[t]{6}{*}{ Salvage treatment } & Re-irradiation & 20 & $15.3 \%$ & 12 & $17.9 \%$ & 8 & $12.5 \%$ & \\
\hline & Chemotherapy (tmz) & 45 & $34.4 \%$ & 24 & $35.8 \%$ & 21 & $32.8 \%$ & \\
\hline & Chemotherapy (other) & 6 & $4.6 \%$ & 3 & $4.5 \%$ & 3 & $4.7 \%$ & \\
\hline & Bevacizumab & 11 & $8.4 \%$ & 5 & $7.5 \%$ & 6 & $9.4 \%$ & \\
\hline & Imatinib & 1 & $0.8 \%$ & 0 & $0.0 \%$ & 1 & $1.6 \%$ & \\
\hline & Dendritic cell vaccination & 1 & $0.8 \%$ & 1 & $1.5 \%$ & 0 & $0.0 \%$ & \\
\hline
\end{tabular}


Table 2 Univariable analysis of potential preditive factors of progression-free survival

\begin{tabular}{|c|c|c|c|c|c|c|c|}
\hline \multirow[b]{2}{*}{ Variable } & \multicolumn{4}{|c|}{ Univariable analysis } & \multicolumn{3}{|c|}{ Multivariable analysis } \\
\hline & $\mathrm{HR}$ & $95 \% \mathrm{Cl}$ & $p$ & Median PFS [m] & $\mathrm{HR}$ & $95 \% \mathrm{Cl}$ & $p$ \\
\hline Age $(<$ vs. $>=$ median of 61 years $)$ & 1.08 & $0.75-1.55$ & 0.69 & 6 vs. 6 & - & - & - \\
\hline Gender ( $m$ vs. f) & 0.68 & $0.46-1.01$ & 0.05 & 6 vs. 9 & 0.57 & $0.35-0.92$ & $0.022(*)$ \\
\hline KPS (< vs. $>=$ median of $70 \%)$ & 0.5 & $0.34-0.72$ & $<0.001\left(^{*}\right)$ & 4 vs. 9 & 0.5 & $0.33-0.78$ & $0.002(*)$ \\
\hline MGMT-status (methylated vs. unmethylated) & 1.46 & $0.97-2.2$ & 0.07 & 9 vs. 6 & 1.61 & $1.03-2.52$ & $0.036(*)$ \\
\hline Localization (other vs. central) & 1.51 & $0.76-3$ & 0.24 & 6 vs. 5 & - & - & - \\
\hline PTV (< vs. $>=$ median of 337 ccm) & 1.13 & $0.79-1.62$ & 0.51 & 7 vs. 6 & - & - & - \\
\hline Subtotal resection or biopsy vs. gross total resection & 0.71 & $0.49-1.02$ & 0.07 & 4 vs. 8 & - & - & - \\
\hline Fractionation regimen (NFRT vs. AHFRT) & 1.01 & $0.95-1.01$ & 0.95 & 7 vs. 6 & - & - & - \\
\hline
\end{tabular}

(*) $p$-value $\leq 0.05$, HR hazard ratio, Cl confidence interval, PFS progression-free survival, KPS Karnofsky performance status, MGMT O-6-methylguanine-DNA methyltransferase, PTV planning target volume, NFRT normofractionated radiotherapy, AHFRT accelerated hyperfractionated radiotherapy

\section{Progression-free survival}

Median PFS was 6 months for the entire cohort (Table 2). For patients treated with NFRT median PFS was 7 months, for patients treated with AHFRT median PFS was 6 months. At 6 months PFS was $56.9 \%$ in the NFRT group and $51.7 \%$ in the AHFRT group. At 12 months PFS was $16.9 \%$ in the NFRT group and $19 \%$ in the AHFRT group, (Fig. 1). There was no difference between both dose regimens in univariable analysis $(p=0.95)$.

\section{Overall survival}

Of 131 patients analyzed 107 had died at the time of analysis (01/2015).
Median OS was 13 months for all patients (Table 3). For patients treated with NFRT median OS was 15 months, for patients treated with AHFRT median OS was 10 months. At 12 months OS was $66 \%$ in the NFRT group and $48.2 \%$ in the AHFRT group. At 24 months OS was $14.7 \%$ in the NFRT group and $16.7 \%$ in the AHFRT group (Fig. 2). There was no difference between both dose regimens in univariable analysis $(p=0.46)$.

\section{Prognostic factors}

Positive predictors of survival in univariable analysis were female gender, higher KPS, MGMT methylation and gross total resection. In multivariable analysis MGMT methylation and gross total resection remained significant

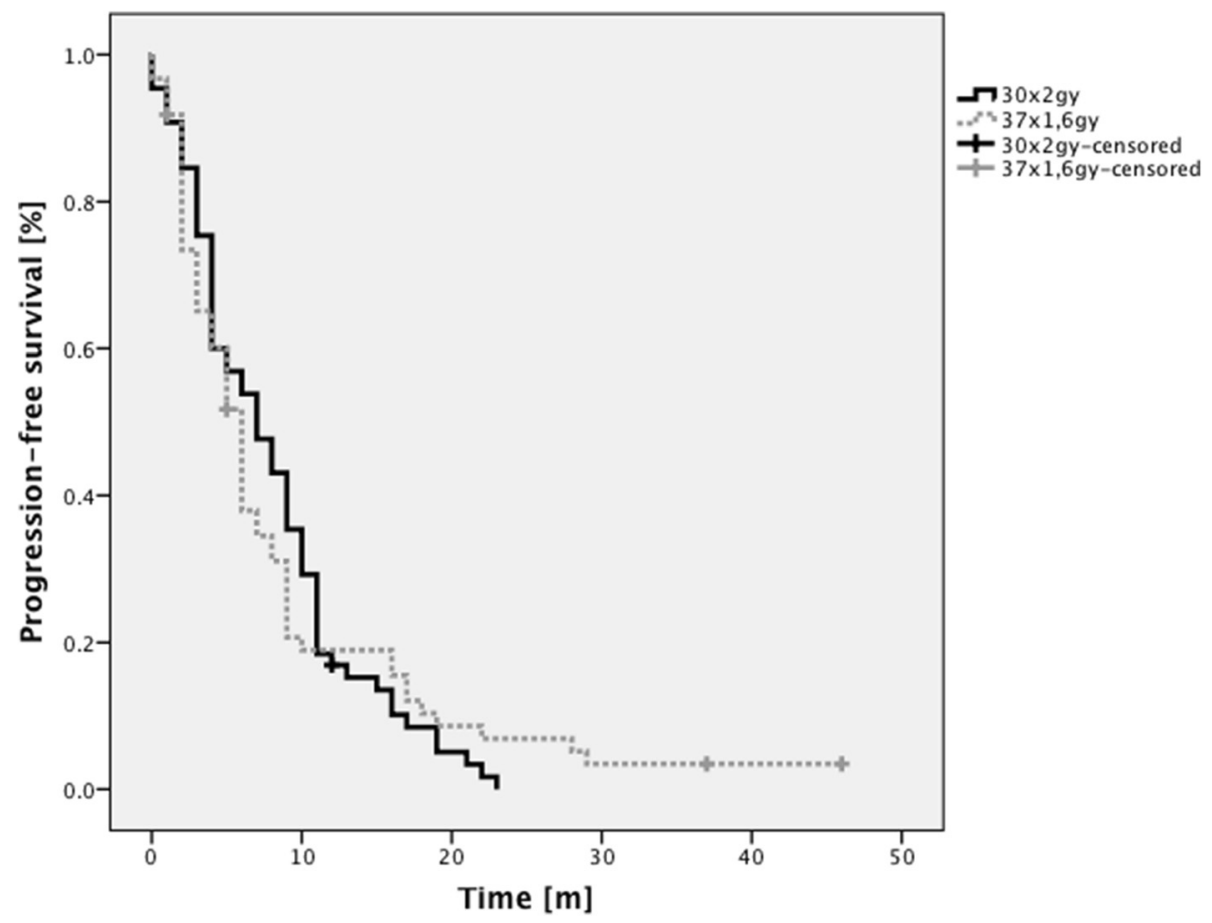

Fig. 1 Kaplan-Meier analysis of PFS rates grouped according to dose regimen. No significant differences were found between both groups 
Table 3 Univariable analysis of potential preditive factors of overall survival

\begin{tabular}{|c|c|c|c|c|c|c|c|}
\hline \multirow[b]{2}{*}{ Variable } & \multicolumn{3}{|c|}{ Univariable analysis } & \multicolumn{4}{|c|}{ Multivariable analysis } \\
\hline & $\mathrm{HR}$ & $95 \% \mathrm{Cl}$ & $p$ & Median OS [m] & $\mathrm{HR}$ & $95 \% \mathrm{Cl}$ & $p$ \\
\hline Age (<vs. $>=$ median of 61 years) & 1.18 & $0.8-1.7$ & 0.4 & 14 vs. 12 & - & - & - \\
\hline Gender ( $m$ vs. $f$ ) & 0.62 & $0.4-0.95$ & $0.028\left(^{*}\right)$ & 11 vs. 16 & 0.64 & $0.38-1.08$ & 0.095 \\
\hline KPS (< vs. > = median of $70 \%)$ & 0.96 & $0.94-0.98$ & $<0.001\left(^{*}\right)$ & 9 vs. 15 & - & - & - \\
\hline MGMT-status (methylated vs. unmethylated) & 1.68 & $1.08-2.61$ & $0.021\left(^{*}\right)$ & 16 vs. 11 & 1.89 & $1.158-3.09$ & $0.011\left(^{*}\right)$ \\
\hline Localization (other vs. central) & 1.71 & $0.83-3.56$ & 0.15 & 13 vs. 13 & - & - & - \\
\hline PTV $(<$ vs. $>=$ median of 337 ccm) & 1.37 & $0.93-2.02$ & 0.11 & 14 vs. 12 & 1.61 & $1-2.6$ & $0.048\left(^{*}\right)$ \\
\hline Subtotal resection or biopsy vs. gross total resection & 0.64 & $0.43-0.95$ & $0.025\left(^{*}\right)$ & 11 vs. 15 & 0.62 & $0.39-0.98$ & $0.041\left(^{*}\right)$ \\
\hline Fractionation regimen (NFRT vs. AHFRT) & 1.16 & $0.79-1.71$ & 0.46 & 15 vs. 10 & - & - & - \\
\hline
\end{tabular}

$\left.{ }^{*}\right) p$-value $\leq 0.05, H R$ hazard ratio, Cl confidence interval, OS overall survival, KPS Karnofsky performance status, MGMT O-6-methylguanine-DNA methyltransferase, PTV planning target volume, NFRT normofractionated radiotherapy, AHFRT accelerated hyperfractionated radiotherapy

predictors, the factor "smaller PTV" became significant in multivariable analysis. Gender and lower KPS were not significant in multivariable analysis.

The fractionation regimen was not a predictor of survival in univariable- or multivariable analysis.

Subgroup analysis according to predictive factors did not reveal any specific group to benefit from either NFRT compared to AHFRT or vice versa (Table 4).

\section{Toxicity}

All patients in both groups completed radiotherapy. All patients scheduled for concurrent chemotherapy (126/131) completed concurrent TMZ. In the normofractionated group seven patients did not complete post-radiotherapy TMZ due to neutropenia or thrombocytopenia. In the hyperfractionated group 3 patients did not complete postradiotherapy TMZ due to neutropenia or thrombocytopenia.

There was no difference in acute toxicity profiles between the two treatment groups. There were seven grade 3 and six grade 4 events in the normofractionated group (grade 3 events: $1 \times$ headache, $2 \times$ neurological, $3 \times$ neutropenia, $1 \times$ thrombocytopenia. Grade 4 events: $2 \times$ neutropenia and $4 \times$ thrombocytopenia).

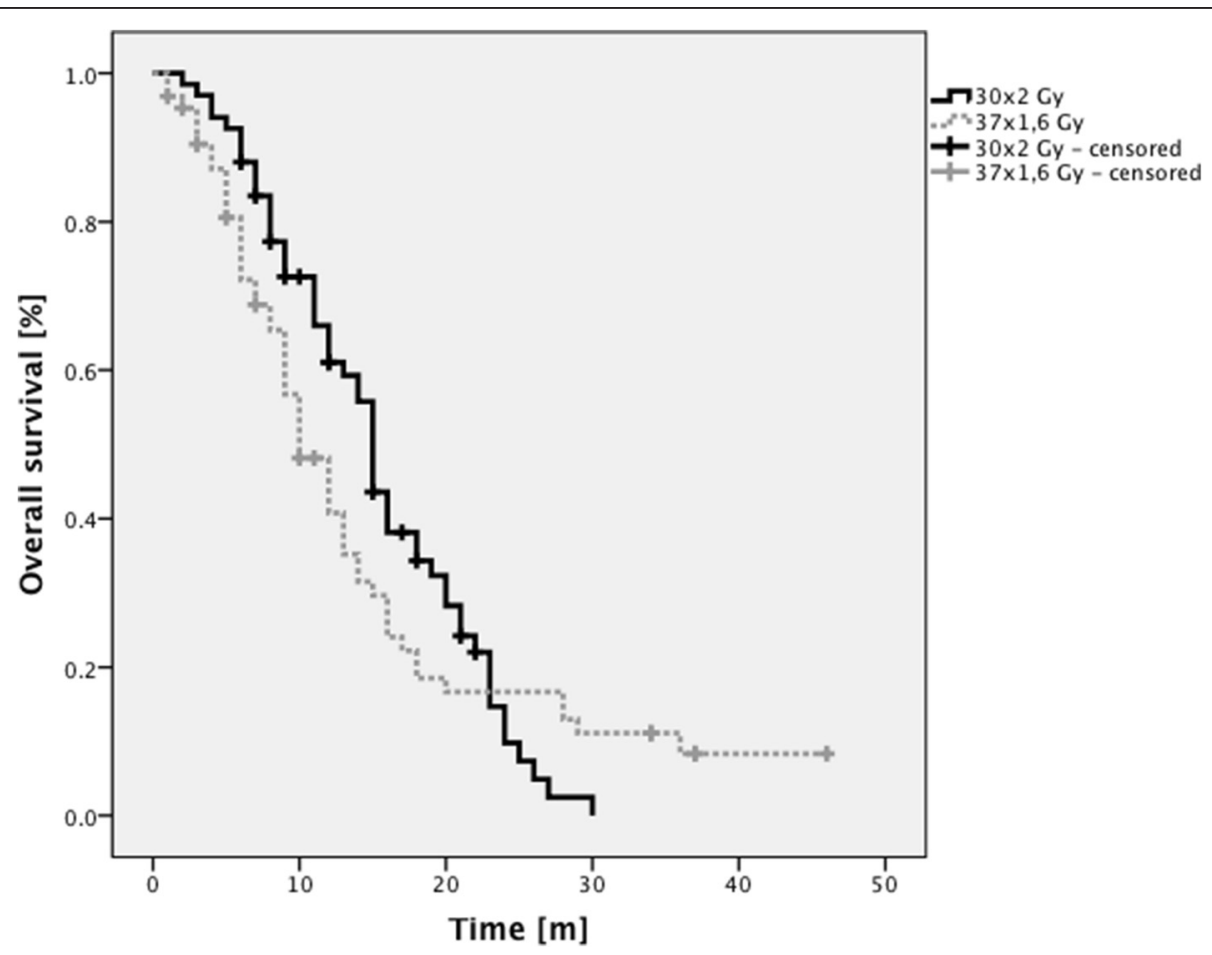

Fig. 2 Kaplan-Meier analysis of OS rates grouped according to dose regimen. No significant differences were found between both groups 
Table 4 Subgroup analysis of potential preditive factors of overall survival did not identify any specific subgroup to benefit from either NFRT compared to AHFRT or vice versa

\begin{tabular}{|c|c|c|c|c|}
\hline & & \multicolumn{3}{|c|}{ Median OS [m] } \\
\hline & & NFRT & AHFRT & $p$ \\
\hline \multicolumn{5}{|l|}{ Variable } \\
\hline \multirow[t]{2}{*}{ Age } & $<$ median of 61 years & 15 & 12 & 0.66 \\
\hline & $>=$ median of 61 years & 15 & 9 & 0.28 \\
\hline \multirow[t]{2}{*}{ Gender } & $\mathrm{m}$ & 14 & 9 & 0.31 \\
\hline & $f$ & 16 & 14 & 0.98 \\
\hline \multirow[t]{2}{*}{ KPS } & $<$ median of $70 \%$ & 12 & 6 & 0.16 \\
\hline & $>=$ median of $70 \%$ & 15 & 13 & 0.67 \\
\hline \multirow[t]{2}{*}{ MGMT-status } & methylated & 16 & 15 & 0.73 \\
\hline & unmethylated & 14 & 9 & 0.09 \\
\hline \multirow[t]{2}{*}{ Localization } & other & 15 & 10 & 0.41 \\
\hline & central & 9 & 17 & 0.44 \\
\hline \multirow[t]{2}{*}{ PTV } & $<$ median of $337 \mathrm{ccm}$ & 15 & 12 & 0.82 \\
\hline & $>=$ median of $337 \mathrm{ccm}$ & 15 & 9 & 0.24 \\
\hline \multirow[t]{2}{*}{ Extent of resection } & Subtotal resection or biopsy & 13 & 8 & 0.14 \\
\hline & gross total resection & 15 & 13 & 0.6 \\
\hline
\end{tabular}

KPS Karnofsky performance status, MGMT 0-6-methylguanine-DNA methyltransferase, PTV planning target volume, NFRT normofractionated radiotherapy, AHFRT accelerated hyperfractionated radiotherapy

In the hyperfractionated group there were two grade 3 events and six grade 4 events (grade 3 events: $1 \times$ neurological, $1 \times$ nausea/vomiting. Grade 4 events: $3 \times$ neutropenia, $3 \times$ thrombocytopenia).

\section{Discussion}

\section{Survival}

Most studies on hyperfractionation and accelerated hyperfractionation stem from the pre-TMZ era, comparability of PFS and OS rates is thus limited. In our study median OS was 13 months for all patients, 15 months for patients treated using NFRT and 10 months for patients treated with AHFRT. Univariable and multivariable analysis did not show significant differences between the fractionation regimens. This is worthwile to know, because an AHFRT-regimen with 3.5 weeks overall treatment time was capable to equalize the OS-results of the classical 6 weeks treatment. Bearing in mind the limited prognosis of these patients the dose-intensified treatment is a clear benefit.

One of the first studies on AHFRT in GBM was published in 1994 by González et al. who used doses of 42-60 Gy in 2 Gy fractions three times a day. Median survival was $8.7 \pm 0.7$ months and no statistically significant differences were found for the four different dose-level groups [13].

Lutterbach et. al. published median OS rates of 8.8 months for 1.5 Gy thrice daily to 54 Gy [14].
In 2001 Prados et al. published survival rates of patients treated with AHFRT \pm DFMO vs. conventional irradiation \pm DFMO with no OS benefit for the experimental groups (8.6-9.8 months) [15].

Werner et al. published the RTOG 83-02 data in 1996, patients received HFRT $(2 \times 1.2$ Gy to doses of $64.8,72,76.8$, or 81.6 Gy $)$ vs. AHFRT $(2 \times 1.6$ Gy to doses of 48 or 54.4 Gy), all groups received concurrent BCNU. Contrary to the other aforementioned studies HFRT patients who had received higher doses of 76.8 and 81.6 Gy showed superior survival compared to the AHFRT groups. The authors found median OS rates between 10.8 and 12.7 months [16].

In 2005 Stupp et al. published data demonstrating a survival benefit for GBM patients that received concurrent Temozolomide with postoperative radiation, with median survival of 14.6 months for patients receiving concurrent therapy versus 12.1 months for patients who received only radiotherapy [7]. This treatment has since become the standard of care for primary GBM and is referred to as the "Stupp regimen" in everyday clinical routine.

OS rates for all patients of 13 months as shown here are comparable to the data published by Stupp et al. and we did not find significant differences in OS between AHFRT and NFRT in our patient collective.

\section{Limitations}

Our study had several limitations. Firstly, the two groups analyzed were not perfectly matched in terms of age. Secondly, the MGMT-status is unknown in approximately $20 \%$ of patients in both treatment groups. Thirdly, no analysis of chronic toxicity was performed due to the intrinsic uncertainties of retrospective analysis. Fourthly, the number of patients analyzed here in both groups might simply be too low to find significant differences in survival between the both regimens. Fifthly, patients with GBM in close proximity to the brainstem were more likely to receive AHFRT, potentially biasing OS rates.

\section{Conclusions}

The role of AHFRT in the TMZ era remains unclear. The potential benefits are a reduction of tumor repopulation as well as reduced late toxicity. Other benefits are immanent; the regimen does significantly shorten hospitalization time in a patient collective with highly impaired life expectancy. We propose that the role of AHFRT + TMZ should be further examined in future prospective trials.

Competing interests

The authors declare that they have no competing interests. 


\section{Authors' contributions}

DK drafted the manuscript, performed statistical analysis and supervised the discussion of the manuscript. JF helped drafting the manuscript, collected data and helped with statistical analysis. HB planned the study and took part in the discussion of the manuscript. AG, PG and SB took part in the discussion of the manuscript. VB planned the study and helped drafting the manuscript. All authors approved the final version of this manuscript.

\section{Received: 11 February 2016 Accepted: 10 May 2016}

\section{Published online: 21 May 2016}

\section{References}

1. Ostrom QT, Gittleman H, Farah P, Ondracek A, Chen Y, Wolinsky Y, Stroup NE, Kruchko C, Barnholtz-Sloan JS. CBTRUS statistical report: Primary brain and central nervous system tumors diagnosed in the United States in 2006-2010. Neuro Oncol. 2013;15 Suppl 2:ii1-56.

2. Paszat L, Laperriere N, Groome P, Schulze K, Mackillop W, Holowaty E. A population-based study of glioblastoma multiforme. Int J Radiat Oncol Biol Phys. 2001;51:100-7.

3. Lacroix M, Abi-Said D, Fourney DR, Gokaslan ZL, Shi W, DeMonte F, Lang FF, McCutcheon IE, Hassenbusch SJ, Holland E, et al. A multivariate analysis of 416 patients with glioblastoma multiforme: prognosis, extent of resection, and survival. J Neurosurg. 2001;95:190-8.

4. Bauchet L, Mathieu-Daude H, Fabbro-Peray P, Rigau V, Fabbro M, Chinot O, Pallusseau L, Carnin C, Laine K, Schlama A, et al. Oncological patterns of care and outcome for 952 patients with newly diagnosed glioblastoma in 2004. Neuro Oncol. 2010;12:725-35.

5. Leu S, von Felten S, Frank S, Vassella E, Vajtai I, Taylor E, Schulz M, Hutter G, Hench J, Schucht P, et al. IDH/MGMT-driven molecular classification of low-grade glioma is a strong predictor for long-term survival. Neuro Oncol. 2013:15:469-79.

6. Keles GE, Anderson B, Berger MS. The effect of extent of resection on time to tumor progression and survival in patients with glioblastoma multiforme of the cerebral hemisphere. Surg Neurol. 1999;52:371-9.

7. Stupp R, Mason WP, van den Bent MJ, Weller M, Fisher B, Taphoorn MJ, Belanger K, Brandes AA, Marosi C, Bogdahn U, et al. Radiotherapy plus concomitant and adjuvant temozolomide for glioblastoma. N Engl J Med. 2005;352:987-96.

8. Malmstrom A, Gronberg BH, Marosi C, Stupp R, Frappaz D, Schultz H, Abacioglu U, Tavelin B, Lhermitte B, Hegi ME, et al. Temozolomide versus standard 6-week radiotherapy versus hypofractionated radiotherapy in patients older than 60 years with glioblastoma: the Nordic randomised, phase 3 trial. Lancet Oncol. 2012;13:916-26.

9. Roa W, Kepka L, Kumar N, Sinaika V, Matiello J, Lomidze D, Hentati D, Guedes de Castro D, Dyttus-Cebulok K, Drodge S, et al. International Atomic Energy Agency Randomized Phase III Study of Radiation Therapy in Elderly and/or Frail Patients With Newly Diagnosed Glioblastoma Multiforme. J Clin Oncol. 2015:33:4145-50.

10. Chen C, Damek D, Gaspar LE, Waziri A, Lillehei K, Kleinschmidt-DeMasters BK, Robischon M, Stuhr K, Rusthoven KE, Kavanagh BD. Phase I trial of hypofractionated intensity-modulated radiotherapy with temozolomide chemotherapy for patients with newly diagnosed glioblastoma multiforme. Int J Radiat Oncol Biol Phys. 2011;81:1066-74.

11. Mizumoto M, Tsuboi K, Igaki H, Yamamoto T, Takano S, Oshiro Y, Hayashi $Y$, Hashii $\mathrm{H}$, Kanemoto A, Nakayama $\mathrm{H}$, et al. Phase I/II trial of hyperfractionated concomitant boost proton radiotherapy for supratentorial glioblastoma multiforme. Int J Radiat Oncol Biol Phys. 2010;77:98-105.

12. Withers HR, Peters LJ, Thames HD, Fletcher GH. Hyperfractionation. Int J Radiat Oncol Biol Phys. 1982:8:1807-9.

13. Gonzalez DG, Menten J, Bosch DA, van der Schueren E, Troost D, Hulshof MC, Bernier J. Accelerated radiotherapy in glioblastoma multiforme: a dose searching prospective study. Radiother Oncol. 1994;32:98-105.

14. Lutterbach J, Weigel P, Guttenberger R, Hinkelbein W. Accelerated hyperfractionated radiotherapy in 149 patients with glioblastoma multiforme. Radiother Oncol. 1999:53:49-52.

15. Prados MD, Wara WM, Sneed PK, McDermott M, Chang SM, Rabbitt J, Page M, Malec M, Davis RL, Gutin PH, et al. Phase III trial of accelerated hyperfractionation with or without difluromethylornithine (DFMO) versus standard fractionated radiotherapy with or without DFMO for newly diagnosed patients with glioblastoma multiforme. Int J Radiat Oncol Biol Phys. 2001;49:71-7.
16. Werner-Wasik M, Scott CB, Nelson DF, Gaspar LE, Murray KJ, Fischbach JA, Nelson JS, Weinstein AS, Curran WJ, Jr. Final report of a phase I/II trial of hyperfractionated and accelerated hyperfractionated radiation therapy with carmustine for adults with supratentorial malignant gliomas. Radiation Therapy Oncology Group Study 83-02. Cancer. 1996;77:1535-43.

17. Niyazi M, Brada M, Chalmers AJ, Combs SE, Erridge SC, Fiorentino A, Grosu AL, Lagerwaard FJ, Minniti G, Mirimanoff RO, et al. ESTRO-ACROP guideline "target delineation of glioblastomas". Radiother Oncol. 2016;118:35-42.

\section{Submit your next manuscript to BioMed Central and we will help you at every step:}

- We accept pre-submission inquiries

- Our selector tool helps you to find the most relevant journal

- We provide round the clock customer support

- Convenient online submission

- Thorough peer review

- Inclusion in PubMed and all major indexing services

- Maximum visibility for your research

Submit your manuscript at www.biomedcentral.com/submit
) BioMed Central 